\title{
Aplicabilidade das metodologias ativas no ensino em saúde à distância: uma revisão integrativa
}

\author{
Applicability of active methodologies in distance learning in health \\ education: an integrative review
}

Aplicabilidad de metodologías activas en la educación a la distancia de la salud: una revisión integrativa

Luciano Ricardo Sfredo ${ }^{1}$, Marina Deina ${ }^{2}$, Carolina Reinert ${ }^{2}$, Ingridy de Souza

Digner ${ }^{2}$, Lucas Zantut ${ }^{2}$.

\footnotetext{
${ }^{1}$ Médico pela Pontifícia Universidade Católica, Cirurgião Geral pelo Hospital Santa Casa de Curitiba e Residente no Serviço de Urologia do Hospital de Clínicas da Universidade Federal do Paraná. Curitiba, Paraná

${ }^{2}$ Acadêmicos do Curso de Graduação em Medicina da Faculdades Pequeno Príncipe. Curitiba, Paraná
}

\section{RESUMO}

Este estudo pretende, através de revisão integrativa, analisar a aplicabilidade de metodologias em um contexto de educação à distância. Para tal, a busca de artigos foi realizada na plataforma

Autor de Correspondência:

*Marina Deina. E-mail: marinadeina@gmail.com 
de pesquisa Google Scholar, utilizando os descritores Educação a Distância, Aprendizado Ativo e Ensino da Saúde. Foram selecionados ao final 12 artigos, cuja análise permitiu a identificação de três categorias que abordam a evolução histórica das metodologias de ensino, ferramentas e sua aplicabilidade ao contexto atual brasileiro e principais dificuldades. O estudo da literatura confirma o protagonismo crescente da inserção dos recursos tecnológicos na educação em saúde e permitiu que fossem identificados os recursos com maior praticidade e viabilidade de aplicação no panorama atual do Brasil. Dentre as Tecnologias Digitais de Informação e Comunicação (TDICs) que permitem a utilização de metodologias como forma de ensino, destacam-se a utilização de narrativas digitais no processo de aprendizado e plataformas virtuais de aprendizagem.

Palavras-Chave: Educação a Distância. Aprendizado Ativo. Educação em Saúde.

\begin{abstract}
This study aims to analyze, through an integrative review, the applicability of methodologies in a context of distance education. The search for articles was carried out on the Google Scholar research platform, using the descriptors Distance Education, Problem-Based Learning, and Health Education. At the end, 12 articles were selected, whose analysis allowed the identification of three categories that address the historical evolution of teaching methodologies, tools, and their applicability to the current Brazilian context, and main difficulties. The study of the literature confirmed the increasingly important role of the insertion of technological resources in health education, and allowed the identification of resources having greater practicality and feasibility of application in the current setting in Brazil. Among the Digital Technologies of Information and Communication allowing the use of methodologies as a way of teaching, the use of digital narratives in the learning process and virtual learning platforms stands out.
\end{abstract}

Keywords: Education, Distance. Problem-Based Learning. Health Education.

\title{
RESUMEN
}

Este estudio tiene como objetivo, a través de una revisión integradora, analizar la aplicabilidad de las metodologías en un contexto de educación a distancia. La búsqueda de artículos se realizó en la plataforma Google Scholar, utilizando los descriptores Educación a distancia, Aprendizaje Basado en Problemas y Educación en Salud. Se seleccionaron 12 artículos, cuyo análisis permitió identificar tres categorías: evolución histórica de metodologías de enseñanza, herramientas y su aplicabilidad al contexto brasileño actual y las principales dificultades. El estudio confirma el papel creciente de la inserción de recursos tecnológicos en la educación en salud y permitió la identificación de recursos con mayor practicidad y factibilidad de aplicación en el panorama actual de Brasil. Entre las Tecnologías Digitales de Información y Comunicación (TDIC) que 
permiten el uso de metodologías como forma de enseñanza, destaca el uso de narrativas digitales en el proceso de aprendizaje y las plataformas virtuales de aprendizaje.

Palabras clave: Educación a Distancia. Aprendizaje Basado en Problemas. Educación en Salud.

\section{INTRODUÇÃo}

Após o surgimento da internet, várias maneiras de conexão e comunicação entre pessoas têm sido criadas e melhoradas. Junto com isso, a ampliação e propagação do conhecimento podem crescer em uma curva exponencial, de maneira tal que tornasse cada vez mais fácil o acesso de sua própria casa a conteúdos antes somente disponíveis em bibliotecas e acervos públicos. ${ }^{1,2,3}$

Com o passar dos anos, essas tecnologias digitais foram se disseminando cada vez mais entre a população e cada vez mais incorporadas aos mais variados âmbitos sociais. ${ }^{1}$ Há vários anos, o processo de ensino em saúde vem passando por modificações em sua estrutura tradicional e grande parte das mudanças que vêm ocorrendo se deve a inserção e utilização de meios digitais e tecnológicos no ensino. ${ }^{1,2,3}$

As tecnologias digitais de comunicação e educação (TDICs) têm alterado a maneira como ocorre a transmissão e aquisição de conhecimento por parte de alunos e professores. Mas para isso, é necessário que as TDICs sejam utilizadas como foco educacional para que seja uma ferramenta útil na construção do conhecimento. ${ }^{1,2}$ Nesse contexto, houve o surgimento do Problem Based Learning (PBL), um novo método de ensino que pretende fazer com que o processo de educação seja baseado no aluno.
Esse método foi pioneiro na McMaster University no Canadá e na Universidade de Maastricht, na Holanda, e posteriormente, foi sendo aplicado em diferentes universidades e países. ${ }^{4}$

De acordo com as Diretrizes Curriculares Nacionais do curso de Medicina, o curso deve ofertar pelo menos parte de sua grade curricular em forma de metodologias ativas de aprendizado. Essas metodologias tiraram o controle total do professor sobre os métodos de aprendizagem de seus alunos, o que faz com que o aluno deixe de ter uma postura somente passiva frente ao estudo e aquisição de novos conhecimentos. ${ }^{4}$ Essa estrutura modificou o processo de ensino e aprendizagem a partir de uma mudança nas estratégias, materiais e meios a serem utilizados para o ensino, bem como a mudança de comportamento e postura de professores e alunos. ${ }^{2,4,5}$

Para que essa mudança se concretize, é necessário utilizar algumas ferramentas digitais no processo de aprendizagem. O presente trabalho objetivou realizar uma revisão integrativa sobre quais são as ferramentas de ensino à distância que utilizem metodologias ativas disponíveis para o meio acadêmico e qual a sua real aplicabilidade no ensino em saúde brasileiro. 


\section{MÉTODO}

Para construção deste estudo, realizamos uma revisão integrativa, que é estruturada em seis etapas. A primeira etapa consiste em estabelecer uma questão de pesquisa, sendo, neste caso, qual a aplicabilidade das metodologias ativas no ensino a distância, de acordo com a literatura.

Apesar das várias ferramentas de ensino a distância, nem todas são passíveis de aplicação prática nos cursos da área da saúde do Brasil. A razão disso é multifatorial, pode envolver tanto fatores econômicos, sociais e culturais. Como fatores econômicos podemos citar a presença ou não de computadores e outros aparelhos digitais nas residências dos alunos, e equipamentos nas universidades que tornem possível a gravação de aulas e interação com os alunos. Já como fatores sociais e culturais, é possível destacar que é necessário que haja um conhecimento por parte dos alunos e professores acerca desses meios e equipamentos digitais para que sejam corretamente utilizados no aprendizado, pois não basta somente o acesso. Essa revisão integrativa busca trazer quais são as ferramentas que melhor se aplicam à realidade brasileira.

Os critérios de inclusão para este projeto foram estudos completamente disponíveis eletronicamente, em português e inglês, publicados sobre o tema a partir de 2008, ou seja, nos últimos 12 anos, realizados com humanos.

Utilizando-se da estratégia de busca foram encontrados inicialmente 153 artigos. Fora do período estipulado eram 38 artigos, restando 115 artigos. Destes, 22 estavam indisponíveis e foram excluídos da pesquisa. Dos 93 artigos restantes, 81 foram excluídos após a leitura dos títulos e resumos. Ao fim da busca, foram selecionados 12 artigos que compuseram a amostra deste trabalho. A seguir, na figura 1, é apresentado um fluxograma que mostra o caminho de seleção dos artigos até a composição da amostra.
Figura 1 - Fluxograma de seleção dos artigos que compõem a amostra.

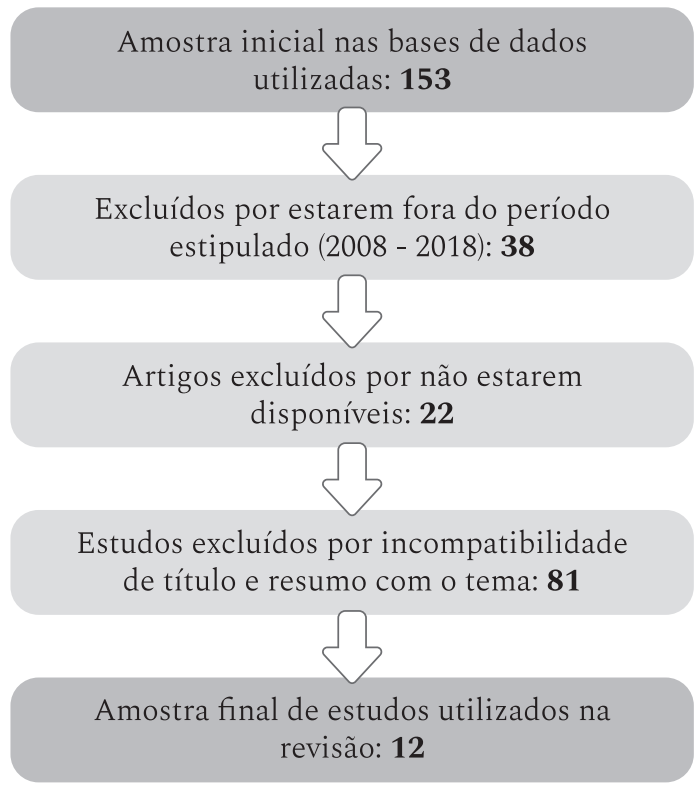

FONTE: os pesquisadores

\section{RESULTADOS}

O quadro 1 apresenta, em ordem cronológica, os estudos selecionados, descrevendo a sua distribuição de acordo com o título, autores, ano de publicação e periódico.

Em uma análise das características gerais dos artigos selecionados é possível perceber que dos doze artigos, apenas dois foram publicados em 2010 ou antes $(16,7 \%)$. Ou outros dez artigos (83,4\%) foram publicados a partir de 2014 , sendo dois $(16,7 \%)$ em 2014; dois (16,7\%) em 2015; um (8,3\%) em 2016; dois (16,7\%) em 2017 e três (25\%) em 2018.

Quanto ao tipo de estudo, oito (66,7\%) artigos são uma revisão de literatura, um $(8,3 \%)$ artigo consistia em um relato de experiência e três (25\%) estudos são de abordagem qualitativa, em que um deles $(8,3 \%)$ consiste em um estudo de caso descritivo com natureza qualitativa. 
Quadro 1 - Artigos organizados conforme título, autores, ano de publicação e periódico.

\begin{tabular}{|c|c|c|c|c|}
\hline № & AUTORES & TÍTULO & $\begin{array}{c}\text { ANO DE } \\
\text { PUBLICAÇÃO }\end{array}$ & PERIÓDICO \\
\hline 1 & Valente JA & $\begin{array}{l}\text { A Comunicação e a Educação baseada } \\
\text { no uso das Tecnologias Digitais de } \\
\text { Informação e Comunicação. }\end{array}$ & 2014 & $\begin{array}{l}\text { Revista UNIFESO } \\
\text { - Humanas e } \\
\text { Sociais }\end{array}$ \\
\hline 2 & $\begin{array}{c}\text { Carvalho RA, Struchiner } \\
\text { M }\end{array}$ & $\begin{array}{l}\text { Análise das Concepções de Saúde na } \\
\text { Produção Acadêmica Brasileira sobre } \\
\text { Educação a Distância na Área da Saúde. }\end{array}$ & 2015 & $\begin{array}{c}\text { Revista Brasileira } \\
\text { de Pesquisa em } \\
\text { Educação em } \\
\text { Ciências }\end{array}$ \\
\hline 3 & Carvalho AAMC. & $\begin{array}{l}\text { O DIGITAL NA EDUCAÇÃO } \\
\text { HUMANISTA EM SAÚDE: } \\
\text { Uma Reflexão Sobre a Inserção e } \\
\text { Potencialidades das Tecnologias Digitais, } \\
\text { no Apoio ao Ensino Técnico na Escola de } \\
\text { Formação em Saúde - EFOS. }\end{array}$ & 2017 & $\begin{array}{c}\text { Observatório } \\
\text { do Cuidado } \\
\text { - Trabalhos de } \\
\text { Conclusão de } \\
\text { Curso. }\end{array}$ \\
\hline 4 & $\begin{array}{l}\text { Cabral HSR, Almeida } \\
\text { KVG. }\end{array}$ & $\begin{array}{l}\text { Problem Based Learning: Aprendizagem } \\
\text { Baseada em Problemas. }\end{array}$ & 2014 & $\begin{array}{l}\text { Revista Interfaces: } \\
\text { Saúde, Humanas e } \\
\text { Tecnologia. }\end{array}$ \\
\hline 5 & Leite BS. & Aprendizagem tecnológica ativa. & 2018 & $\begin{array}{l}\text { Rev. Inter. Educ. } \\
\text { Sup. Campinas. }\end{array}$ \\
\hline 6 & $\begin{array}{l}\text { Farias PAM, Martin } \\
\text { ALAR, Cristo CS. }\end{array}$ & $\begin{array}{l}\text { Aprendizagem Ativa na Educação em } \\
\text { Saúde: Percurso Histórico e Aplicações. }\end{array}$ & 2015 & $\begin{array}{l}\text { Rev. bras. educ. } \\
\text { med. }\end{array}$ \\
\hline 7 & $\begin{array}{c}\text { Gimeniz-Paschoal SR, } \\
\text { Belei RA, Nascimento } \\
\text { EN. }\end{array}$ & $\begin{array}{l}\text { História curricular dos cursos de } \\
\text { graduação da área da saúde. }\end{array}$ & 2008 & $\begin{array}{l}\text { Rev. História da } \\
\text { Educação }\end{array}$ \\
\hline 8 & $\begin{array}{l}\text { Reis ZSN, Melo MCB, } \\
\text { Corrêa EJ, Pereira AK, } \\
\text { Santos DB, Alves HJ. }\end{array}$ & $\begin{array}{l}\text { Tecnologias digitais para o ensino } \\
\text { em saúde: relato de experiências e a } \\
\text { convergência para o Projeto } \mathrm{AVAs}^{21} \text {. }\end{array}$ & 2016 & $\begin{array}{c}\text { Revista de } \\
\text { Saúde Digital } \\
\text { e Tecnologias } \\
\text { Educacionais. }\end{array}$ \\
\hline 9 & $\begin{array}{l}\text { Silva JB, Andrade MH, } \\
\text { Oliveira RR, Sales GL, } \\
\text { Alves FRV. }\end{array}$ & $\begin{array}{l}\text { Tecnologias digitais e metodologias } \\
\text { ativas na escola: o contributo do Kahoot } \\
\text { para gamificar a sala de aula. }\end{array}$ & 2018 & Rev. Thema. \\
\hline 10 & $\begin{array}{l}\text { Silva ASR, Oliveira LR, } \\
\text { Cavalcante LE, Rolim } \\
\text { RM, Souza LF. }\end{array}$ & $\begin{array}{l}\text { Metodologias ativas de ensino e } \\
\text { aprendizagem em educação a distância } \\
\text { em saúde. }\end{array}$ & 2018 & $\begin{array}{c}\text { Revista de } \\
\text { Saúde Digital } \\
\text { e Tecnologias } \\
\text { Educacionais. }\end{array}$ \\
\hline 11 & $\begin{array}{c}\text { Palácio MAV, Ciannella } \\
\text { D, Struchiner M. }\end{array}$ & $\begin{array}{l}\text { Narrativas Digitais e Aprendizagem: um } \\
\text { panorama a partir do ensino da saúde. }\end{array}$ & 2017 & $\begin{array}{l}\text { Rev Eletron Comun } \\
\text { Inf Inov Saúde. }\end{array}$ \\
\hline 12 & Marin MJS et al. & $\begin{array}{l}\text { Aspectos das fortalezas e fragilidades } \\
\text { no uso das metodologias ativas de } \\
\text { aprendizagem. }\end{array}$ & 2010 & $\begin{array}{l}\text { Rev. bras. educ. } \\
\text { med. }\end{array}$ \\
\hline
\end{tabular}


Todos os estudos ocorreram em Instituições de Ensino Superior com cursos da área da saúde. Os pesquisadores eram todos profissionais de cursos da área da saúde e da área de educação e os participantes das pesquisas foram estudantes de cursos da área da saúde. Desta forma, a reunião de tais estudos contempla de forma abrangente o conteúdo disponível para o ensino em saúde no que tange ao aprendizado dos estudantes.

Os eixos temáticos foram variados, abordando avaliações de métodos de estudo, avaliação dos métodos de aprendizagem, viabilidade dos recursos tecnológicos disponíveis, treinamento profissional para o ensino a distância e panorama atual de utilização de recursos online no Brasil.

Quanto ao periódico de publicação dos estudos, dois artigos (16,7\%) foram publicados na Revista Brasileira de Educação Médica e dois artigos (16,7\%) foram publicados na Revista de Saúde Digital e Tecnologias Educacionais, enquanto os demais foram publicados cada um em um respectivo periódico, incluindo a Revista Interfaces: Saúde, Humanas e Tecnologia, Revista Internacional de Educação Superior, Revista Brasileira de Pesquisa em Estudos em Ciências, Revista Thema, Revista UNIFESO - humanas e sociais, Revista Eletrônica de Comunicação, Informação e Inovação em Saúde e Revista História da Educação.

\section{DISCUSSÃo}

Os artigos selecionados permitiram a classificação dos temas em três categorias, que fornecem o subsídio para a discussão do estudo, sendo elas: “evolução histórica das metodologias de ensino", "ferramentas e sua aplicabilidade ao contexto atual brasileiro" e "principais dificuldades".

\section{Evolução histórica das metodologias de ensino}

Desde a época de Atenas clássica, Platão utilizava-se de espaços abertos para lecionar seus discípulos, que possuíam liberdade para refletir, opinar, conversar e experimentar. A ideia de haver uma instituição que concentrasse a disseminação do conhecimento por parte dos sábios surgiu entre os anos de 384 e 383 a.C. ${ }^{6}$

Vários anos depois, o americano Abraham Flexner propôs um modelo de ensino similar ao que estava sendo utilizado na Alemanha, em que o curso de medicina era dividido em dois ciclos: ciclo básico e ciclo clínico, sendo que o primeiro seria responsabilidade das universidades da época, e o segundo seria responsabilidade exclusiva das instituições hospitalares. A ideia não apresentou bons resultados no aprendizado dos alunos e este foi considerado um modelo de formação ultrapassado. ${ }^{6}$

No Brasil, o ensino iniciou-se por meio dos jesuítas, sob a influência da religião católica, assim como também se deu em vários outros países. As primeiras escolas públicas surgiram no período de 1759 a 1827, sob a influência de ideias iluministas segundo a estratégia do despotismo esclarecido. ${ }^{6}$

A educação no século XX foi resultado das ideias de uma série de pensadores, filósofos e estudiosos do tema, e o ensino passa a ser direcionado à necessidade de autonomia do estudante. Nas décadas de 70 e 80 , ganhou força um movimento de pedagogia crítica, que compartilhava a ideia de que a escola deveria ser um local em que a realidade fosse problematizada. Foi justamente a conclusão de que era necessário ampliar a autonomia do estudante que contribuiu para o surgimento das metodologias ativas. Estas metodologias têm a função de formar profissionais independentes, críticos e formadores de opinião. Porém, é importante lembrar que a combinação de metodologias é a estratégia que até 
hoje trouxe melhores resultados no aprendizado. O uso de uma não necessariamente exclui o uso de outra metodologia associada. ${ }^{7}$

\section{Ferramentas e sua aplicabilidade no contexto atual brasileiro}

Um dos principais desafios da inclusão das Tecnologias Digitais de Informação e Comunicação (TDICs) ativas na formação de profissionais da saúde é que a maioria das salas de aula das universidades brasileiras ainda mantêm o formato antigo, em que o professor encontra-se no mais alto nível hierárquico e atua como detentor do conhecimento, bem como a maior parte das atividades são realizadas com caneta e papel. ${ }^{1,2,8,9}$

Em 1998, foi criado o conceito de "estar junto virtual", que consiste na troca de ideias, questionamento, desafio e fornecimento da informação necessária para que um determinado grupo de alunos possa avançar, ou seja, não é somente uma conexão via rede. Os alunos inseridos neste contexto se utilizam da comunicação e troca de experiências na resolução de problemas ou elaboração de um projeto. Para isso, é necessário que o especialista (professor) esteja junto com os aprendizes por meio de alguma plataforma online para que seja capaz de auxiliá-los na resolução de seus problemas, vivenciando as situações e percebendo onde encontram-se as dificuldades do grupo. ${ }^{1}$

O ensino a distância possui a vantagem de que algumas interações realizadas via internet permitem alguns aprendizados mais difíceis de serem realizados presencialmente. Apesar disso, ainda existem muitas críticas acerca do $\mathrm{EaD}$, com alegações de que a postura passiva dos estudantes permanece a mesma, assim como a centralização do conhecimento e das regras de ensino ainda permanece na figura do professor. ${ }^{1,2}$ É importante ressaltar que em cursos da área da saúde, se priorizam tutorias realizadas por profissionais formados na área que está sendo estudada, o que apresenta-se como uma das maiores dificuldades logísticas apontadas pelas universidades brasileiras. ${ }^{1,8}$

Vários outros recursos são apontados como facilitadores do aprendizado no ensino a distância. Um desses recursos é a plataforma conhecida como Ambiente Virtual de Ensino-Aprendizagem (AVA), que permite que os professores passem a atuar efetivamente como tutores nas atividades realizadas via plataforma. Esta plataforma pode abrigar diferentes recursos de aprendizagem, como fóruns de discussão entre alunos ou alunos e professores, salas de bate-papo, webconferências, quando necessário, vídeo aulas, compartilhamento de arquivos, podcasts em áudio, casos simulados e arquivos multimidiáticos. Além disso, torna-se possível realizar correções de atividades e feedbacks. Ressalta-se também a importância da utilização destes recursos na entrega de trabalhos que antes eram realizados na forma física, em papel, de maneira que a implementação deste recurso resulte em um menor impacto ambiental. 3,8

Quanto à formação de professores capacitados para trabalhar com o ensino em saúde a distância, os profissionais recebem treinamento e formação específica para atuarem online, de acordo com suas áreas de atuação. Esses cursos buscam o desenvolvimento de competências para atuar como mediadores do ensino-aprendizagem, e não detentores, e também buscam capacitar os profissionais para que sejam capazes de implementar ações pedagógicas com o uso de metodologias ativas. $^{10}$

O AVA também permite que o aluno seja capaz de decidir de maneira autônoma os horários que deseja realizar as atividades de acordo com sua preferência e tempo disponível, respeitando seu ritmo individual. Isto é importante para o desenvolvimento 
da responsabilidade do aluno e otimização de seu aprendizado, processo de memorização e raciocínio. ${ }^{3,8}$

É importante destacar que os principais modelos de educação ativa são baseados nos princípios da andragogia e seus principais pilares são o autodirecionamento, a aplicação de conhecimento na solução de problemas, a motivação para aprender, o respeito às experiências prévias dos estudantes e o reconhecimento das relações entre os motivos da aprendizagem e o papel social do futuro profissional. ${ }^{8}$

Outra ferramenta aplicável no ensino a distancia é a utilização de narrativas digitais, que podem ser realizadas de diversas formas e que são capazes de organizar experiências e promover a reflexão. A estrutura narrativa permite que o conhecimento do estudante seja organizado em começo, meio e fim, o que faz com que seu entendimento sobre a experiência seja aumentado. No ensino em saúde atual, existe uma defesa de ressignificação da formação profissional, fazendo que esta seja mais voltada ao paciente e seu contexto social. As reflexões da narrativa propiciam um protagonismo também do aluno em sua formação. A narrativa também é capaz de gerar reflexões do estudante quanto a sua postura ou a dos demais envolvidos na experiência a partir da leitura de suas ações. Porém, é preciso lembrar que para que esse processo tenha sucesso, é necessário que o processo de construção da narrativa seja crítico, associado a um movimento reflexivo. ${ }^{11}$

\section{Principais dificuldades}

Dentre as principais dificuldades encontradas na busca deste estudo, destaca-se o fato de que, apesar dessas tecnologias de ensino em saúde terem revolucionado a $\mathrm{EaD}$, muitas atividades que vêm sendo realizadas à distância podem ser caracterizadas como uma imitação da educação presencial. ${ }^{2}$

Além disto, a literatura aponta que uma das principais reclamações dos alunos se dá pelo motivo de que a mudança de metodologia se dá de forma abrupta. No ensino básico da maioria das escolas brasileiras, o método tradicional de ensino prevalece e em grande parte dos casos é realizado de maneira exclusiva. Já quando o aluno ingressa em uma universidade que se utiliza de metodologias ativas, a transição é feita de uma maneira muito rápida, em que os alunos se sentem perdidos na busca e organização do novo conhecimento. ${ }^{12}$

Atualmente, os jogos online são uma forma de entretenimento popular entre boa parte dos estudantes e podem ser grandes aliados no aprendizado, principalmente por atrair o aluno a aprender e fazer o que lhe traz prazer. Porém, o conhecimento de bases que promovam uma integração do ensino e conhecimento de forma gamificada é muito limitado por parte dos profissionais, que acabam queixando-se de plataformas que sejam capazes de realizar um sistema de feedbacks imediatos e inclusão de jogos e maneiras mais descontraídas de promover o ensino. ${ }^{5,9}$

No Brasil, um grande desafio do Ministério da Saúde e das universidades tem sido encontrar uma maneira de levar e promover a informação de modo crítico e reflexivo para profissionais que atuam em regiões muito distantes dos centros urbanos ou isoladas. ${ }^{10}$

\section{CONCLUSÕES}

O estudo da literatura confirma o protagonismo crescente dos recursos tecnológicos em nosso meio, bem como permite a identificação de alguns dos elementos que podem estar associados a um melhor desempenho no papel de educar em saúde.

Dentre as dificuldades e limitações da utilização de meios tecnológicos no ensino em saúde, foi 
observado que o acesso à internet ainda não é feito de maneira completa por todos os estados brasileiros. Existem muitas localidades que não possuem acesso à internet ou o têm de maneira precária. Além disso, a mudança abrupta na metodologia de estudo que passa de um ensino básico tradicional para um superior com metodologias ativas é uma queixa frequente na literatura.

Como meios tecnológicos aplicáveis à realidade brasileira e que se utilizam de metodologias ativas, a literatura destaca a narrativa, em especial na forma escrita por meio de portfólios, o uso das TDICs, de maneira que permita a interação professor-aluno à distância, plataformas virtuais como o Ambiente Virtual de Ensino-Aprendizagem (AVA), capaz de atender a uma variedade de requisitos para que se possa estabelecer uma metodologia ativa mesmo à distância, além da utilização cada vez mais frequente de jogos que promovam a ampliação do conhecimento, apesar de seu uso ainda ser bastante limitado.

\section{REFERÊNCIAS}

1. Valente JA. A Comunicação e a Educação baseada no uso das Tecnologias Digitais de Informação e Comunicação. Rev. UNIFESO - Humanas e Sociais [Internet]. 2014 [Acesso em 2020 maio 15]; 1(1): 141-166. Disponível em: http://www.revista.unifeso.edu.br/index.php/ revistaunifesohumanasesociais/articl\%20e/view/17/2\%204.

2. Carvalho RA, Struchiner M. Análise das Concepções de Saúde na Produção Acadêmica Brasileira sobre Educação a Distância na Área da Saúde. Revista Brasileira de Pesquisa em Educação em Ciências [Internet]. 2015 [Acesso em 2020 maio 15]; 15(3): 457-476. Disponível em: https://periodicos. ufmg.br/index.php/rbpec/article/view/4324.

3. Carvalho AAMC. O DIGITAL NA EDUCAÇÃO HUMANISTA EM SAÚDE: Uma Reflexão Sobre a Inserção e Potencialidades das Tecnologias Digitais, no Apoio ao Ensino Técnico na Escola de Formação em Saúde - EFOS. Porto Alegre. TCC [Especialização em Acompanhamento, Monitoramento e Avaliação na Educação em Saúde Coletiva] - Universidade Federal do Rio Grande do Sul. 2017. Disponível em: https://repositorio. observatoriodocuidado.org/handle/handle/1214.

4. Cabral HSR, Almeida KVG. Problem Based Learning: Aprendizagem Baseada em Problemas. Revista Interfaces: Saúde, Humanas e Tecnologia [Internet]. 2014 [Acesso em 2020 maio 15]; 2(esp). Disponível em: http://interfaces. leaosampaio.edu.br/index.php/revista-interfaces/article/ view/35.

5. Leite BS. Aprendizagem tecnológica ativa. Rev. Inter. Educ. Sup. Campinas [Internet]. 2018 [Acesso 2020 maio 14]; 4(3): 580-609. Disponível em: https://periodicos.sbu. unicamp.br/ojs/index.php/riesup/article/view/8652160.

6. Farias PAM, Martin ALAR, Cristo CS. Aprendizagem Ativa na Educação em Saúde: Percurso Histórico e Aplicações. Rev. bras. educ. med. [Internet]. 2015 [Acesso 2020 maio 15]; 39(1): 143-150. Disponível em: https://www. scielo.br/scielo.php?script=sci_abstract\&pid=S0100$55022015000100143 \& \operatorname{lng}=$ pt\&nrm=iso\&tlng=pt.

7. Gimeniz-Paschoal SR, Belei RA, Nascimento EN. História curricular dos cursos de graduação da área da saúde. Revista História da Educação [Internet]. 2008 [Acesso 2020 maio 14]; 12(24): 101-120. Disponível em: https://seer. ufrgs.br/asphe/article/view/29228.

8. Reis ZSN, Melo MCB, Corrêa EJ, Pereira AK, Santos DB, Alves HJ. Tecnologias digitais para o ensino em saúde: relato de experiências e a convergência para o Projeto AVAS21. Revista de Saúde Digital e Tecnologias Educacionais [Internet]. 2016 [Acesso em 2020 maio 14]; 1(1): 69-76. Disponível em: http://periodicos.ufc.br/resdite/ article/view/4685.

9. Silva JB, Andrade MH, Oliveira RR, Sales GL, Alves FRV. Tecnologias digitais e metodologias ativas na escola: o contributo do Kahoot para gamificar a sala de aula. Revista Thema [Internet]. 2018 [Acesso em 2020 maio 14]; 15(2): 780-791. Disponível em: http://revistathema.ifsul.edu. br/index.php/thema/article/view/838/791.

10. Silva ASR, Oliveira LR, Cavalcante LE, Rolim RM, Souza LF. Metodologias ativas de ensino e aprendizagem em educação à distância em saúde. Revista de Saúde Digital e Tecnologias Educacionais [Internet]. 2018 [Acesso em 2020 maio 15]; 3(1): 15-26. Disponível em: http://www.periodicos. ufc.br/resdite/article/view/40059. 
11. Palácio MAV, Ciannella D, Struchiner M. Narrativas Digitais e Aprendizagem: um panorama a partir do ensino da saúde. Reciis - Rev Eletron Comun Inf Inov Saúde [Internet]. 2017 [Acesso em 2020 maio 15]; 11(2): 1-15. Disponível em: https://www.reciis.icict.fiocruz.br/index. php/reciis/article/view/1111.

12. Marin MJS et al. Aspectos das fortalezas e fragilidades no uso das metodologias ativas de aprendizagem. Rev. bras. educ. med. [Internet]. 2010 [Acesso em 2020 maio 15]; 34(1): 13-20. Disponível em: https://www.scielo.br/ scielo.php?pid=S0100 $-55022010000100003 \&$ script $=$ sci abstract\&tlng=pt. 